University of Nebraska - Lincoln

DigitalCommons@University of Nebraska - Lincoln

John R. Hardy Papers

Research Papers in Physics and Astronomy

3-15-1973

Static Equilibrium Conditions for a Rigid-Ion Crystal

\author{
L. L. Boyer \\ University of Nebraska - Lincoln \\ John R. Hardy \\ University of Nebraska - Lincoln
}

Follow this and additional works at: https://digitalcommons.unl.edu/physicshardy

Part of the Physics Commons

Boyer, L. L. and Hardy, John R., "Static Equilibrium Conditions for a Rigid-Ion Crystal" (1973). John R. Hardy Papers. 16.

https://digitalcommons.unl.edu/physicshardy/16

This Article is brought to you for free and open access by the Research Papers in Physics and Astronomy at DigitalCommons@University of Nebraska - Lincoln. It has been accepted for inclusion in John R. Hardy Papers by an authorized administrator of DigitalCommons@University of Nebraska - Lincoln. 


\title{
Static Equilibrium Conditions for a Rigid-Ion Crystal*
}

\author{
L. L. Boyer ${ }^{\dagger}$ and J. R. Hardy \\ Behlen Laboratory of Physics, University of Nebraska, Lincoln, Nebraska 68508
}

(Received 25 September 1972)

\begin{abstract}
The equilibrium requirements for a static ionic crystal impose a number of constraints on the short-range forces which become increasingly more important when one is dealing with complex structures. These constraints are presented and discussed for a general rigid-ion lattice, and a specific discussion is presented for the wurtzite structure.
\end{abstract}

In a lattice composed of rigid ions we have two types of forces: the long-range Coulomb interactions between the constituent ions and the shortrange forces acting between relatively close neighbors which are essential to prevent the structure from collapsing. If we assume central short-range forces then, for any structure, we must ensure that the static lattice is in equilibrium, both with respect to any macroscopic strain and also with respect to relative motion of the constituent sublattices (i.e., internal strain). For the more complex crystal structures this leads to a number of 
constraints on the short-range forces which can be effectively used to determine certain short-range force constants. ${ }^{1}$ Surprisingly, these constraints have not been explicitly included in some earlier lattice-dynamical calculations. ${ }^{2-4}$

It is true that these authors do not make the assumption of two-body forces and our arguments are not directly applicable to their work. However, the use of arbitrary tensor force fields to simulate covalent bonding is questionable, since the resultant potential functions are often not rotationally invariant. One can overcome this problem by postulating terms in the potential which resist bond bending, but if this is done then the equilibrium conditions will still provide constraints on the force constants which must be satisfied.

Strictly speaking, anharmonic effects may modify the constraints. However, these modifications are usually small and are neglected.

A general discussion of these constraints (staticequilibrium conditions) is given by Born and

Huang, ${ }^{\mathbf{5}}$ but they do not separately treat the Coulomb and short-range interactions, and therefore their treatment is not very useful when applied to ionic crystals. On the other hand, Keffer and Portis ${ }^{6}$ have treated these Coulomb contributions, but they do not explicitly relate their results to stability conditions involving the short-range interactions. Here we present in detail the static-equilibrium conditions for a general rigid-ion crystal.

Consider a lattice of point charges each labeled by a combined index $(l, k)$, where $l$ denotes a particular cell and $k$ labels the ions within that cell. Let the lattice vectors be given by

$$
\overrightarrow{\mathrm{x}}(l)=\overrightarrow{\mathrm{x}}\left(l_{1}, l_{2}, l_{3}\right)=l_{1} \overrightarrow{\mathrm{a}}_{1}+l_{2} \overrightarrow{\mathrm{a}}_{2}+l_{3} \overrightarrow{\mathrm{a}}_{3},
$$

where $\vec{a}_{1}$, $\vec{a}_{2}$, and $\vec{a}_{3}$ are the primitive lattice vectors and $l_{1}, l_{2}$, and $l_{3}$ are integers. Basis vectors are denoted by $\overrightarrow{\mathrm{x}}(k)$. For convenience we establish the following notation:

$$
\begin{aligned}
& \overrightarrow{\mathrm{x}}(l, k) \equiv \overrightarrow{\mathrm{x}}(l)+\overrightarrow{\mathrm{x}}(k), \quad \overrightarrow{\mathrm{x}}\left(l, k k^{\prime}\right) \equiv \overrightarrow{\mathrm{x}}(l, k)-\overrightarrow{\mathrm{x}}\left(0, k^{\prime}\right), \\
& \overrightarrow{\mathrm{x}}\left(k k^{\prime}\right) \equiv \overrightarrow{\mathrm{x}}\left(0, k k^{\prime}\right),
\end{aligned}
$$

where $l=0$ means that $l_{1}=l_{2}=l_{3}=0$. We also adopt this notation for $\overrightarrow{\mathbf{r}}$ and $\overrightarrow{\mathrm{u}}$, where $\overrightarrow{\mathbf{r}}(l, k)$ is the position of the $(l, k)$ ion displaced from its equilibrium position $\overrightarrow{\mathbf{x}}(l, k)$ by an amount $\overrightarrow{\mathrm{u}}(l, k)$.

For any pairwise potential, the energy per unit cell is given by

$$
U=\frac{1}{2} \sum_{l k k^{\prime}}{ }^{\prime} \psi\left(r\left(l, k k^{\prime}\right)\right),
$$

where $\psi\left(r\left(l, k k^{\prime}\right)\right)$ is the potential energy (Coulomb and short range) of the bond between the $(l, k)$ and $\left(0, k^{\prime}\right)$ ions, and $r\left(l, k k^{\prime}\right)$ is the distance between them. The prime on the summation sign in Eq. (1) indicates that the $(0, k k)$ terms are omitted, and the factor $\frac{1}{2}$ avoids double counting.

Expanding $U$ to first order in the displacements, we have

$$
\begin{aligned}
U=\frac{1}{2} \sum_{l k k^{\prime}}{ }^{\prime}( & \psi\left(x\left(l, k k^{\prime}\right)\right)+\psi^{\prime}\left(x\left(l, k k^{\prime}\right)\right) \\
& \left.\times \sum_{\alpha} \frac{x_{\alpha}\left(l, k k^{\prime}\right)}{x\left(l, k k^{\prime}\right)} u_{\alpha}\left(l, k k^{\prime}\right)\right) .
\end{aligned}
$$

For a homogeneous deformation the displacements may be written as a sum of the contributions due to internal and external strains $\left[u_{\alpha}(k)\right.$ and $\left.s_{\alpha \beta}\right]$ :

$$
u_{\alpha}(l, k)=u_{\alpha}(k)+\sum_{\beta} s_{\alpha \beta} x_{\beta}(l, k) .
$$

Substituting Eq. (3) into (2), we have

$$
\begin{aligned}
U=\frac{1}{2} \sum_{l k k^{\prime}} \cdot[\psi( & \left.x\left(l, k k^{\prime}\right)\right)+\psi^{\prime}\left(x\left(l, k k^{\prime}\right)\right) \sum_{\alpha} \frac{x_{\alpha}\left(l, k k^{\prime}\right)}{x\left(l, k k^{\prime}\right)} \\
& \left.\times\left(u_{\alpha}\left(k k^{\prime}\right)+\sum_{\beta} s_{\alpha \beta} x_{\beta}\left(l, k k^{\prime}\right)\right)\right] .
\end{aligned}
$$

The stability conditions $\partial U / \partial u_{\alpha}\left(k^{\prime}\right)=0$ and $\partial U / \partial s_{\alpha \beta}=0$ give

$$
-\sum_{k l}^{\prime} \psi^{\prime}\left(x\left(l, k k^{\prime}\right)\right) \frac{x_{\alpha}\left(l, k k^{\prime}\right)}{x\left(l, k k^{\prime}\right)}=0
$$

and

$$
\frac{1}{2} \sum_{l k k^{\prime}}{ }^{\prime} \psi^{\prime}\left(x\left(l, k k^{\prime}\right)\right) \frac{x_{\alpha}\left(l, k k^{\prime}\right) x_{\beta}\left(l, k k^{\prime}\right)}{x\left(l, k k^{\prime}\right)}=0 .
$$

Equation (5) ensures that the crystal be stable against the internal strain $\overrightarrow{\mathrm{u}}\left(k^{\prime}\right)$, while Eq. (6) ensures stability against the external strain $s_{\alpha \beta}$. Evidently Eq. (5) is equivalent to the statement that the net force on any given sublattice in the crystal is zero, while Eq. (6) requires that the total macroscopic stress is zero.

Writing the Coulomb and short-range contributions to $\psi$ separately, Eqs. (5) and (6) become

$$
\sum_{k l}^{\prime} \phi^{\prime}\left(x\left(l, k k^{\prime}\right)\right) \frac{x_{\alpha}\left(l, k k^{\prime}\right)}{x\left(l, k k^{\prime}\right)}+F_{\alpha}\left(k^{\prime}\right)=0
$$

and

$$
\frac{1}{2} \sum_{l k k^{\prime}}{ }^{\prime} \phi^{\prime}\left(x\left(l, k k^{\prime}\right)\right) \frac{x_{\alpha}\left(l, k k^{\prime}\right) x_{\beta}\left(l, k k^{\prime}\right)}{x\left(l, k k^{\prime}\right)}+f_{\alpha \beta}=0,
$$

where $\phi$ denotes the short-range potential and $F_{\alpha}\left(k^{\prime}\right)$ and $f_{\alpha \beta}$ are derived from the Coulomb contributions to $\psi$. Explicitly, $\boldsymbol{F}_{\alpha}\left(k^{\prime}\right)$ is the force on the $k^{\prime}$ ion due to all the Coulomb interactions and $f_{\alpha \beta}$ is the Coulomb contribution to the macroscopic stress. Notice that for the Coulomb potential $\phi_{c}(x)$ we have $\phi_{c}^{\prime}(x)=-\phi_{c}(x) / x$, which leads to the result that $\sum_{\alpha} f_{\alpha \alpha}=-U_{C}$, where $U_{C}$ is the Coulomb energy of the unit cell. Thus, for cubic crystals, Eq. (8) reduces to a single equilibrium condition which is identical to that obtained by minimizing the unit- 
cell energy with respect to a uniform dilation if the Coulomb energy is written in the usual Madelung form $U_{C}=-\alpha_{m} e^{2} / r$, where $\alpha_{m}$ is the appropriate Madelung constant.
More generally, the Coulomb contributions to $F_{\alpha}\left(k^{\prime}\right)$ and $f_{\alpha \beta}$ may be put into a rapidly convergent form using the Ewald technique ${ }^{7}$ with the following results:

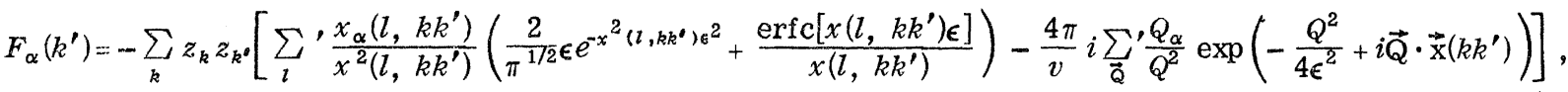

$$
\begin{aligned}
& f_{\alpha \beta}=-\frac{1}{2} \sum_{k k^{\prime}} z_{k} z_{k^{\prime}}\left\{\sum_{l}, \frac{x_{\alpha}\left(l, k k^{\prime}\right) x_{\beta}\left(l, k k^{\prime}\right)}{x^{2}\left(l, k k^{\prime}\right)}\left(\frac{2}{\pi^{1 / 2}} \epsilon e^{-x^{2}\left(l, k k^{\prime}\right) \epsilon^{2}}+\frac{\operatorname{erfc}\left[x\left(l, k k^{\prime}\right) \epsilon\right]}{x\left(l, k k^{\prime}\right)}\right)\right. \\
& \left.+\frac{4 \pi}{v} \sum_{\overrightarrow{\mathrm{Q}}} \cdot\left[\frac{\delta_{\alpha \beta}}{Q^{2}}-\frac{Q_{\alpha} Q_{\beta}}{Q^{2}}\left(\frac{2}{Q^{2}}+\frac{1}{2 \epsilon^{2}}\right)\right] \exp \left(-\frac{Q^{2}}{4 \epsilon^{2}}+i \overrightarrow{\mathrm{Q}} \cdot \overrightarrow{\mathrm{x}}\left(k k^{\prime}\right)\right)\right\},
\end{aligned}
$$

where $z_{k}$ is the charge of the $k$ th ion, $\vec{Q}$ is a reciprocal-lattice vector, erfc is the complementary error function, $v$ is the volume of the unit cell, and $\varepsilon$ is a convergence parameter. The prime on the summation over $l$ means that for $k=k^{\prime}$ the $l=0$ term is omitted, and the prime on the summation over $\vec{Q}$ means that the $\vec{Q}=0$ term is omitted for all values of $k$ and $k^{\prime}$.

Equations (9) and (10) are easily programmed for the computer to accommodate an arbitrary crystal lattice, and for the proper choice of the convergence parameter $(1 / \epsilon$ is approximately equal to the lattice constant) the sums over both real and reciprocal space are rapidly convergent. We have computed $\overrightarrow{\mathrm{F}}(k)$ and $f_{\alpha \beta}$ for the wurtzite structure for comparison with the results of Keffer and Portis. ${ }^{6}$

The wurtzite structure is specified by three primitive lattice vectors

$$
\begin{aligned}
& \vec{a}_{1}=r_{0}(\sqrt{2}, 0,0), \quad \vec{a}_{2}=r_{0}(\sqrt{2}, \sqrt{6}, 0), \\
& \vec{a}_{3}=\frac{4}{3} r_{0}(0,0, \sqrt{3}),
\end{aligned}
$$

and four basis vectors $\overrightarrow{\mathrm{x}}(k), k=1,2,3,4$,

$$
\overrightarrow{\mathbf{x}}(1)=(0,0,0),
$$

$$
\begin{aligned}
& \overrightarrow{\mathrm{x}}(2)=\frac{1}{6} r_{0}(3 \sqrt{2}, \sqrt{6}, \sqrt{3}), \\
& \overrightarrow{\mathrm{x}}(3)=\frac{1}{6} r_{0}(3 \sqrt{2}, \sqrt{6}, 4 \sqrt{3}), \\
& \overrightarrow{\mathrm{x}}(4)=\frac{1}{6} r_{0}(0,0,-3 \sqrt{3}) .
\end{aligned}
$$

We take the charge of the ions to be $z_{1}=z_{3}=+e$ and $z_{2}=z_{4}=-e$, where $e$ is the electronic charge.

The resultant sublattice forces are along the preferred $\alpha=3$ direction with

$$
\begin{aligned}
& F_{3}(1)=F_{3}(3)=C, \\
& F_{3}(2)=F_{3}(4)=-C,
\end{aligned}
$$

where $C=0.0198173\left(e^{2} / r_{0}^{2}\right)$. The Coulomb tensor $f_{\alpha \beta}$ is diagonal with

$$
\begin{aligned}
& f_{11}=f_{22}=1.2372665\left(e^{2} / r_{0}\right), \\
& f_{33}=1.3159370\left(e^{2} / r_{0}\right) .
\end{aligned}
$$

The values of $f_{\alpha \alpha}$ are positive with $f_{33}>f_{11}$, indicating that the Coulomb forces tend to compress or collapse the structure, and this compression is strongest along the preferred $\alpha=3$ direction. These results are in agreement with those of Keffer and Portis. ${ }^{6}$
* Research sponsored by the Air Force Office of Scientific Research, Air Force Systems Command, USAF, under Grant No. AFOSR 70-1926. The United States Government is authorized to reproduce and distribute reprints for Governmental purposes notwithstanding any copyright notation hereon.

${ }^{\dagger}$ Now at Solid State and Materials Laboratory, Princeton University, Princeton, N. J.

${ }^{1}$ L. L. Boyer and J. R. Hardy, Bull. Am. Phys. Soc. 17, 246 (1972).
${ }^{2}$ M. A. Nusimovici and J. L. Birman, Phys. Rev. 156, 925 (1967).

${ }^{3}$ J. Sakurai, R. A. Cowley, and G. Dolling, J. Phys. Soc. Jap. 28, 1426 (1970).

${ }^{4}$ E. R. Cowley, Phys. Rev. B 3, 2733 (1971).

${ }^{5} \mathrm{M}$. Born and K. Huang, Dynamical Theory of Crystal Lattices (Oxford U. P., New York, 1954), Eqs. 11.13 and 11.14.

${ }^{6}$ F. Keffer and A. M. Portis, J. Chem. Phys. 27, 675 (1957).

${ }^{7}$ P. P. Ewald, Ann. Phys. (Leipz.) 64, 253 (1921). 